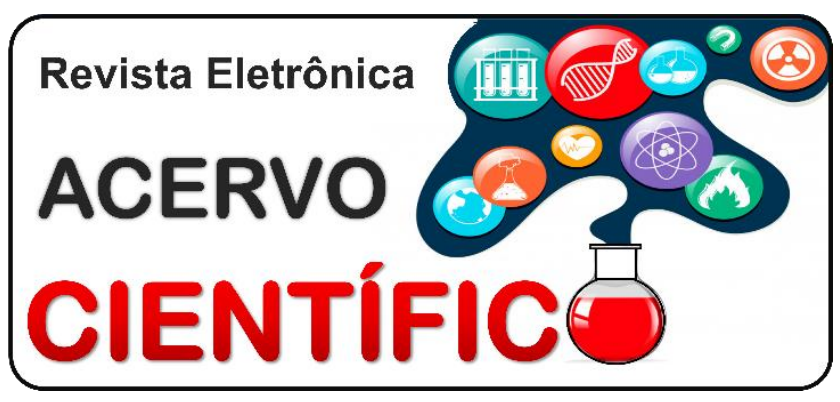

\section{REVISÃO BIBLIOGRÁFICA}

Recebido em: 9/2020

Aceito em: 10/2020

Publicado em: 2/2021

\title{
A segurança do paciente em âmbito cirúrgico
}

\author{
Patient safety in surgery \\ Seguridad del paciente em el ámbito quirúgico
}

Gabriela Ferreira da Silva ${ }^{1}$, Leandro Fellet Miranda Chaves ${ }^{1}$, Geovana Torres de Souza ${ }^{1}$, Caroline Silva de Araujo Lima², lago José Cunha Silva ${ }^{3}$.

\begin{abstract}
Resumo: Revisão integrativa da literatura com o objetivo de identificar os controles de qualidade na redução de riscos ao paciente no centro cirúrgico. A busca ocorreu em três bases de dados eletrônicas, abrangendo o período de 2010 a 2019, com os descritores: "Segurança do Paciente", "Centros Cirúrgicos", "Controle de Qualidade" e "Hospitalização". Para isso, foram abordadas discussões sobre: (i) erros de medicação, (ii) infecção de sítio cirúrgico e hospitalização, (iii) erro de incisão cirúrgica e (iv) esquecimento de material cirúrgico no paciente. O estudo permitiu a reflexão da importância da equipe multiprofissional em relação aos quesitos necessários para a realização de uma cirurgia segura, reduzindo, com isso, a possibilidade de ocorrência de danos aos pacientes. Além disso, a redução dos riscos e dos danos e a inserção de boas práticas favorecem a efetividade dos cuidados da equipe de modo seguro, principalmente em tempos de pandemia. Portanto, a aplicação na íntegra do checklist de cirurgia segura é fundamental para a redução de danos ao paciente cirúrgico.
\end{abstract}

Palavras-chave: Segurança do paciente, Centros cirúrgicos, Controle de qualidade.

\begin{abstract}
Integrative review of the literature with the objective of identifying quality controls in the reduction of risks to the patient in the surgical center. The search occurred in three electronic databases, covering the period from 2010 to 2019, with the descriptors: "Patient Safety", "Surgical Centers", "Quality Control" and "Hospitalization". For this purpose, discussions were addressed: (i) medication errors, (ii) surgical site infection and hospitalization, (iii) surgical incision error and (iv) forgetfulness of surgical material in the patient. The study allowed reflection on the importance of the multiprofessional team in relation to the necessary requirements for safe surgery, thus reducing the possibility of damage to patients.In addition, the reduction of risks and damages and the insertion of good practices favour the effectiveness of team care in a safe way, especially in times of pandemic. Therefore, the full application of the safe surgery checklist is fundamental for the reduction of damage to the surgical patient.
\end{abstract}

Key words: Patient safety, Surgical centers, Quality control.

\footnotetext{
1 Universidade Federal de Juiz de Fora (UFJF), Juiz de Fora - MG.

*E-mail: gabriela.ferreira@medicina.ufjf.br

${ }^{2}$ Faculdade Dinâmica do Vale Piranga (FADIP), Ponte Nova - MG.

${ }^{3}$ Universidade de Pernambuco (UPE), Recife - PE.
} 
Resumen: Revisión de la literatura integradora con el objetivo de identificar los controles de calidad en la reducción de riesgos para el paciente en el centro quirúrgico. La búsqueda se realizó en tres bases de datos electrónicas, que abarcan el período comprendido entre 2010 y 2019, con los descriptores: "Seguridad del paciente", "Centros quirúrgicos", "Control de calidad" y "Hospitalización". Con este fin, se abordaron los debates: i) errores de medicación, ii) infección del sitio quirúrgico y hospitalización, iii) error en la incisión quirúrgica y iv) olvido del material quirúrgico en el paciente. El estudio permitió reflexionar sobre la importancia del equipo multiprofesional en relación con los requisitos para una cirugía segura, reduciendo así la posibilidad de que se produzcan daños a los pacientes. Además, la reducción de riesgos y daños y la inserción de buenas prácticas favorecen la eficacia de la atención en equipo de manera segura, especialmente en tiempos de pandemia. Por lo tanto, la aplicación completa de la lista de verificación de cirugía segura es fundamental para la reducción de los daños al paciente quirúrgico.

Palabras clave: Seguridad del paciente, Centros quirúrgicos, Control de calidad.

\section{INTRODUÇÃO}

Segundo Martins FZ e Dall'Agnol CM (2016), o centro cirúrgico (CC) é um setor restrito da instituição hospitalar onde são executados procedimentos anestésico-cirúrgicos, diagnósticos e terapêuticos. Ele é considerado um cenário de alto risco com situações envolvendo risco de vida e/ou sequela de pacientes onde os processos de trabalho constituem-se em práticas complexas e interdisciplinares.

Nesse sentido, foi criado em 2009 pela Organização Mundial da Saúde (OMS), a lista de verificação (checklist) "Cirurgias Seguras salvam vidas" para Segurança do Paciente. A iniciativa foi uma resposta à Resolução 55.18 da Assembleia Mundial da Saúde, que recomendou à OMS e aos Estados-Membros a maior atenção possível ao problema da segurança do paciente. O objetivo da lista de verificação (checklist) é realizar uma checagem de pré-requisitos no intuito de se evitar erros e eventos adversos. Este checklist é composto de três etapas, sendo elas: (1) antes da indução anestésica, o período após a indução e (2) antes de iniciar a cirurgia e os períodos durante ou imediatamente após o fechamento da ferida operatória e por fim (3) antes do paciente sair da sala (MINISTÉRIO DA SAÚDE, 2009).

Os protocolos dos checklist adaptados ao contexto são mais susceptíveis de serem utilizados e sustentados na prática. A fidelidade e a sustentabilidade aumentam quando os protocolos da lista de verificação podem ser integrados na prática profissional diária. A incorporação rotineira de protocolos da lista de verificação é influenciada por fatores que promovem ou inibem a participação dos médicos (FERRAZ EM, 2013).

O checklist utiliza estratégias com potencial para melhorar o desenvolvimento da equipe, com um foco particular na comunicação, objetivando mitigar o potencial de erros durante a cirurgia. Seu propósito como uma ferramenta de segurança é de criar um diálogo entre os membros da equipe. Tal estratégia visa o uso de uma abordagem consistente na comunicação interdisciplinar da equipe (ROSCANI ANCP, et al., 2015).

À vista disso, o presente estudo tem por objetivo revisar os dados disponíveis na literatura no que se refere à segurança do paciente no Centro Cirúrgico. $O$ trabalho procura enfatizar quais os erros preveníveis mais frequentes nesse ambiente, atentando-se aos pretextos que corroboram para a sua ocorrência, bem como às estratégias plausíveis para mitigá-los. Não obstante, levando em consideração a pandemia do COVID-19 e as preocupações quanto à transmissão deste patógeno dentro do hospital, será abordado também o panorama de cuidados especiais referentes à contenção do novo coronavírus no CC.

\section{REVISÃO BIBLIOGRÁFICA}

\section{Os erros mais frequentes no centro cirúrgico}

As cirurgias e as hospitalizações, independente da condição financeira e de sua localidade, sejam urbanas ou rurais, ocorrem em números expressivos: estima-se que, a cada ano, sejam realizados mais de 200 milhões de procedimentos cirúrgicos ao redor do mundo (HAYNES AB, et al., 2009). Devido a 
gigantesca expressão numérica, é notável que os CC sejam susceptíveis a diversos resultados desfavoráveis, sendo a maioria deles evitáveis (MANRIQUE BT, et al., 2015). Muitos desses eventos decorrem de problemas de comunicação entre a equipe cirúrgica, da falta de protocolos de segurança eficazes ou do não seguimento destes. Sendo assim, o assunto é de extrema relevância, considerando-se os possíveis prejuízos ao paciente e as implicações éticas e legais aos profissionais responsáveis. (HAYNES AB, et al., 2009)

Incidentes relacionados às medicações são caracterizados como quaisquer erros evitáveis em processos de saúde que possam causar dano aos pacientes, desde a caracterização dos medicamentos, comunicação entre os profissionais, monitoramento e uso. Tais equívocos podem gerar ônus profundos para os pacientes e seus familiares, além de atribuir custos extras ao tratamento e potencialmente à reabilitação desse paciente. As principais falhas concentram-se na administração do fármaco incorreto (48\%) e na superdosagem do medicamento (38\%), seguidos pela escolha inapropriada da via de administração (8\%), subdosagem (4\%) e omissões (2\%). A justificativa para esses achados pode estar associada à variedade de medicamentos com frascos e nomes parecidos, além da troca de etiquetas de seringas e erros no cálculo da dose medicamentosa indicada (DHAWAN I, et al., 2017).

No que tange às medidas preventivas, recomenda-se a implantação de protocolos padronizados almejando a consolidação de práticas seguras entre os profissionais. Uma dessas estratégias é a adoção de etiquetas com cores padronizadas por grupo terapêutico, que constitui um método visual de prevenção de erros. Além disso, estimula-se a dupla checagem antes do preparo da medicação, bem como a identificação clara das seringas com nome do medicamento, concentração e via de administração, a fim de mitigar a troca de agulhas no momento de aplicação do medicamento. Não obstante é de suma importância que medicamentos que requerem vias de administração diferentes sejam armazenados separadamente (ISMP, 2018).

Outro assunto primordial são as infecções pós-operatórias do sítio cirúrgico, que acometem mais de $5 \%$ dos pacientes, apesar de evitável na maior parte dos casos. Esse desdobramento é altamente expressivo, visto que amplia o tempo médio de internação em 9,7 dias e aumenta de 2 a 11 vezes o risco de mortalidade. Nesse sentido, recomenda-se a administração de antibiótico profilático parenteral até uma hora antes do início da cirurgia, antissepsia da pele com solução a base de álcool, controle glicêmico perioperatório, regulação da temperatura e manutenção da oxigenação tecidual adequada. Atualmente desencoraja-se a continuação da antibioticoterapia profilática após o fechamento da pele em pacientes com baixo risco de infecção do sítio cirúrgico. Além disso, a aplicação de curativos de pressão negativa tem demonstrado resultados promissores pela associação a menores taxas de contaminação e aumento da perfusão vascular ao redor da incisão, corroborando para adequada cicatrização do corte (FIELDS AC, et al., 2020).

Um fator que é pouco levado em consideração nas pesquisas do que compete às infecções de sítio cirúrgico são os prováveis e possíveis problemas que podem vir a ocorrer após a alta do paciente. Grande parte dos pacientes recebe alta precoce, seja pelo elevado custeio de recursos e insumos gastos na internação, seja pela questão da demanda de leitos. Martins T, et al. (2018) informam em seu artigo que $61 \%$ das infecções de sítio cirúrgico foram detectadas após o período hospitalar, o que comprova a necessidade de vigilância e acompanhamento dos recém operados. Além da necessidade de a própria equipe envolvida na cirurgia fazer uma busca ativa em manter contato com o paciente, torna-se essencial que a unidade de saúde da comunidade onde o recém operado reside se disponha a entrar em contato com o próprio paciente e com sua família na finalidade de fortalecer e solidificar a observação do quadro geral e específico do paciente, pois dessa forma as chances de infecções de sítio cirúrgico se tornam menores e caso ocorram serão identificadas de forma precoce, reduzindo as chances de agravo (MARTINS T, et al., 2018)

No que diz respeito ao sítio cirúrgico incorreto, existem as variáveis de erro de lado a ser operado, local de operação e troca de implante. Além disso, existem séries de casos em que um paciente foi submetido a um procedimento cirúrgico que deveria ser realizado em outro indivíduo. Nos Estados Unidos, encontrou-se uma média de 0,09 erros a cada 10.000 procedimentos cirúrgicos (HEMPEL S, et al., 2015).

Com o propósito de evitar esses desfechos, os checklists prezam pela confirmação oral do nome do paciente, do procedimento a ser realizado e do sítio cirúrgico no momento de admissão do paciente na sala 
cirúrgica. Ademais, recomenda-se a marcação do local de incisão antes do paciente ser anestesiado com material resistente à antissepsia (HAYNES AB, et al., 2009).

O esquecimento de utensílios cirúrgicos dentro do paciente também constitui um desfecho frequente, com média de 1,32 eventos a cada 10.000 cirurgias. O material cirúrgico esquecido mais corriqueiramente são as compressas cirúrgicas. Os danos causados por esse erro podem ser assintomáticos ou podem manifestar-se muitos anos após a cirurgia, até que o paciente relate algum desconforto ou realize um exame de rotina que demonstre a presença desse corpo estranho. Como forma de evitar esse tipo de ocorrência, deve ser normativa que a equipe realize uma contagem oral dos materiais depois do procedimento para a certificação de que todas as compressas ou qualquer outro instrumento utilizado tenham sido retirados do paciente (HEMPEL S, et al., 2015).

\section{Antissepsia pré-cirúrgica e o manejo de fontes de contaminação}

A antissepsia cirúrgica das mãos é um procedimento indispensável para a atuação da equipe multidisciplinar no ambiente cirúrgico. O objetivo é remover a sujidade e oleosidade da pele, bem como eliminar a microbiota transitória e reduzir a microbiota residente, além de propiciar efeito residual na pele do profissional (ANVISA, 2010). Fazer a antissepsia cirúrgica, muitas vezes, é considerado um ato simples e sem grande importância. Todavia, a higienização é de grande importância, sendo uma medida essencial para evitar que o paciente seja contaminado por microrganismos provenientes de outras pessoas e também para proteger os profissionais da saúde de possíveis doenças.

A técnica deverá ser realizada em um lavabo cirúrgico, com o uso de escovas e esponjas descartáveis, de uso individual e que contém soluções anti sépticas degermantes à base de gluconato de clorexidina $2 \%$ ou Polivinilpirrolidona lodado 10\% (PVPI) (ANVISA, 2010). Para a realização da técnica são recomendados de 15 movimentos para as unhas e 15 para cada área da pele. De acordo com os Órgãos Reguladores e as Comissões Internas de Controle de Infecções Hospitalares $(\mathrm{CCIH})$ o lavabo deve ser localizado próximo à entrada da sala de operação, que deve possuir torneiras com mecanismos que dispensem o uso das mãos, com sistema de água fria e quente.

Todavia, dentre os erros mais frequentes no momento da antissepsia encontram-se o emprego incorreto da técnica de escovação, paramentação inadequada e utilização de materiais oxidados. A escovação ideal implica em retirada de adornos como anéis, brincos e cordões, devido ao potencial de contaminação através desses objetos. Quanto à paramentação, avental cirúrgico, luva estéril, touca, máscara, propé e óculos são equipamentos rotineiros que servem para proteção não apenas do paciente mas também do profissional (MORAES ACFG, 2011).

Outro ponto a se considerar na questão de segurança do paciente, são as infecções e contaminações levadas de fora para dentro da sala cirúrgica; vários protocolos já são seguidos com a finalidade de evitar tais acontecimentos, como por exemplo a assepsia e antissepsia dos cirurgiões, anestesistas, enfermeiros e instrumentadores, além do uso de vestimenta limpa e adequada para o procedimento a ser realizado. No entanto, alguns objetos levados constantemente para o centro cirúrgico são bolsas, telefones celulares, chaves de automóveis e acessórios que, por anteriormente terem tido contato com o mundo externo, livre de assepsia e repleto de microrganismos, são potenciais transportadores de micróbios. Um estudo realizado em um hospital filantrópico de Recife, analisou swabs umedecidos de caldo enriquecedor de 50 telefones celulares de profissionais que atuam em cirurgia e foram encontrados os seguintes microrganismos em 48 deles: Estafilococus coagulase-negativa, Bacillus Subtilis e Micrococcus sp (CUNHA CBC, 2016)

\section{A influência da atual pandemia do Coronavírus no âmbito cirúrgico}

O surgimento da pandemia ocasionada pela COVID-19 modificou a rotina da população mundial e trouxe vários questionamentos e dúvidas acerca do vírus e de sua propagação, tomando local de destaque tanto na mídia quanto nas temáticas de estudos científicos. É de conhecimento geral que a disseminação do vírus ocorre de forma acelerada e trouxe muitos impactos sociais e econômicos a todo o mundo, e não seria diferente no tópico tangente à realização de cirurgias na pandemia e em seus desdobramentos de prevenção e transmissão (DO BU EA, et al., 2020). 
A fim de orientar o comportamento das equipes cirúrgicas, o Colégio Brasileiro de Cirurgiões recomenda medidas de segurança como forma de lidar com cirurgias no período da pandemia (CORREIA MITD, et al., 2020). Nesse sentido, as salas cirúrgicas e de recuperação pós anestésica precisaram se adaptar e criar um ambiente exclusivo para casos suspeitos ou confirmados de COVID-19. Ao fim das cirurgias, os EPIs e materiais não reutilizáveis são descartados em sacos identificados como risco biológico vermelho e a sala é higienizada com solução a base de hipoclorito de sódio (BENÍTEZ CY, et al., 2020).

As recomendações feitas pelo Colégio Brasileiro de Cirurgiões cita por exemplo a sugestão de que as cirurgias eletivas, isto é, aquelas que podem ser postergadas sem causar dano ao prognóstico do paciente, devem ser agendadas novamente para momentos mais oportunos, na intenção de liberar o acesso de ventiladores mecânicos e evitar contaminação hospitalar. Dessa forma, apenas as atividades essenciais devem ser realizadas durante a pandemia, de caráter de urgência e emergência. Similarmente, é orientado que devem ser utilizadas salas específicas para a realização das operações, priorizando a desinfecção rigorosa da sala e dos materiais utilizados. Toda equipe deve estar orientada sobre o uso de equipamentos de proteção individual (EPI), assim como os visitantes, que devem ser reduzidos e limitados, a fim de diminuir o tráfego de pessoas e objetos e garantir ao paciente menor contato com potenciais transmissores da doença (CORREIA MITD, et al., 2020).

Sabe-se que o controle da disseminação do vírus é pautado pelo uso da máscara, higienização das mãos e distanciamento social, de forma que o respeito a essas recomendações se faz ainda mais necessário no ambiente hospitalar (BATISTA J, et al., 2020).

Em associação com as medidas protetivas para pacientes e profissionais da saúde na pandemia de coronavírus, os tópicos e questionamentos relacionados à realização de cirurgias eletivas nos setores oncológicos também foram levantados. Embora os pacientes oncológicos sejam tidos como grupo de risco elevado para o covid-19, postergar a intervenção cirúrgica pode ser ainda mais prejudicial devido à progressão da doença, devendo cada caso ser analisado à parte, considerando-se todos os riscos envolvidos (JI C, et al., 2020). O seguimento de orientações nesse caráter são valorosas pois ditam medidas a serem seguidas que tem como intenção primordial o reconhecimento da magnitude do problema e o enfrentamento dele nas melhores condições possíveis a todos os envolvidos

\section{Desafios documentados da implantação do checklist}

A implementação do checklist na sala de operação reforça a segurança do paciente, melhora a comunicação entre os profissionais e reduz o número de complicações pós-operatórias, incluindo óbitos. Foi demonstrado que a adesão do checklist elaborado pela Organização Mundial da Saúde reduziu a mortalidade de $1,5 \%$ para $0,8 \%$, e a taxa de complicação pós cirúrgica, de $11 \%$ para $7 \%$ (HAYNES AB, et al., 2009). Não obstante, o risco de infecção cirúrgica após a adoção deste diminuiu em até $43 \%$, com redução de complicações mais significativa em países em desenvolvimento (PRATES CG, et al., 2018).

Um dos desafios para a adesão de um novo checklist de segurança cirúrgica baseia-se na necessidade da mudança de comportamento por parte de toda a equipe envolvida, bem como a compreensão genuína de como esta ferramenta pode impactar a estadia do paciente no hospital. Nesse sentido, médicos e enfermeiros precisam se adaptar ao novo sistema proposto. Uma etapa importante é fazer com que esses profissionais se habituem ao protocolo, e sintam que o checklist faz parte do time cirúrgico, de maneira que tenham consciência do quão importante é o preenchimento deste, a fim de valorizar e tornar mais real a segurança do paciente (BERGS J, et al., 2015).

A implementação do processo por si só pode agir como uma barreira e causar resistência ao checklist pelos surgimentos de dúvidas e controvérsias. Sendo assim, seu conteúdo precisa ser apoiado por evidências científicas, deve ser escrito de maneira clara e propor estratégias relevantes para a situação. Quanto à relutância da equipe em aceitar um novo protocolo, é importante incentivá-los através da demonstração de estudos clínicos com resultados positivos e atividades práticas (BERGS J, et al., 2015).

Outra ocorrência relatada pelas equipes como um possível agravante da resistência ao protocolo de checagem é a necessidade de confirmar identidade e procedimento mais de uma vez, além de perguntas 
específicas a respeito de perda sanguínea e condições de respiração, o que possivelmente causa aumento na ansiedade e insegurança do paciente. O ponto mais esquecido sobre a checagem foi a confirmação dos membros da equipe cirúrgica, justificado pela baixa rotatividade e grande familiaridade entre o time - o que fez com que esse passo fosse considerado menos importante (BERGS J, et al., 2015). Por outro lado, um estudo brasileiro demonstrou maior adesão ao checklist nos tópicos associados a desfechos potencialmente devastadores para o paciente, como por exemplo a verificação do sítio cirúrgico correto (RIBEIRO HCTC, et al., 2017).

\section{CONSIDERAÇÕES FINAIS}

O estudo permitiu a reflexão sobre a alta incidência de eventos potencialmente danosos ao paciente no centro cirúrgico, realçando o número significativo de complicações evitáveis. Ratifica-se a importância de uma comunicação assertiva entre os membros da equipe cirúrgica, visto que muitos desses desdobramentos podem ser prevenidos. No que diz respeito ao checklist do centro cirúrgico, percebe-se que o instrumento auxilia na segurança do paciente e estimula a comunicação entre a equipe envolvida no processo cirúrgico. Além disso, a redução dos riscos e dos danos e a inserção de boas práticas favorecem a efetividade dos cuidados da equipe de modo seguro, principalmente em tempos de pandemia onde a saúde e integridade da equipe e do paciente são de extrema importância.

\section{REFERÊNCIAS}

1. BATISTA J, et al. Estratégia multimodal para higiene das mãos em hospitais de campanha de COVID-19. Revista Brasileira de Enfermagem, 2020.

2. BENÍTEZ CY, et al. Adaptação a um cenário sem precedente: cirurgia durante o surto de COVID-19. Revista do Colégio Brasileiro de Cirurgiões, 2020.

3. BERGS J, et al. Barriers and facilitators related to the implementation of surgical safety checklists: a systematic review of the qualitative evidence. BMJ Quality and Safety, 2015; 24(12):776-86.

4. BRASIL. Agência Nacional de Vigilância Sanitária (ANVISA). Segurança do Paciente - Higienização das Mãos. Brasília, 2010. Disponível em:<http://www.anvisa.gov.br/servicosaude/manuais/paciente_hig_maos.pdf> Acesso em: outubro de 2020.

5. BRASIL. Ministério da Saúde Cirurgias seguras salvam vidas Rio de Janeiro: 2009. Pan-Americana da Saúde; Ministério da Saúde; Agência Nacional de Vigilância.

6. CORREIA MITD, et al. Os cirurgiões e a pandemia do COVID-19. Revista do Colégio Brasileiro de Cirurgiões, 2020.

7. CUNHA CBC. Avaliação microbiológica dos aparelhos celulares dentro do bloco cirúrgico - Avaliação de um hospital beneficente em Pernambuco. Dissertação (Mestrado) - Universidade Federal de Pernambuco, CCS. Programa de Pós-Graduação em Cirurgia. Recife, 2016.

8. DO BU, E. A. et al, Representações e ancoragens sociais do novo coronavírus e do tratamento da COVID-19 por brasileiros. Estudos de psicologia, 2020.

9. DHAWAN I, et al. Erros de medicação em anestesia: inaceitável ou inevitável? Revista Brasileira de Anestesiologia, 2017.

10. FERRAZ EM. A cirurgia segura. Uma exigência do século XXI. Revista do Colégio Brasileiro de Cirurgiões, 2009.

11. FIELDS AC, et al. Preventing Surgical Site Infections Looking Beyond the Current Guidelines. Journal of the American Medical Association, 2020;323(11):1087-1088.

12. HAYNES AB, et al. A Surgical Safety Checklist to Reduce Morbidity and Mortality in a Global Population. The New England Journal of Medicine,2003; 360(5): 491-499

13. HEMPEL S, et al., Wrong-Site Surgery, Retained Surgical Items, and Surgical Fires: A Systematic Review of Surgical Never Events. Journal of the American Medical Association Surgery, 2015; 150(8):796-805.

14. ISMP - Instituto para Práticas Seguras no uso de Medicamentos, Brasil. Segurança no uso de medicamentos em cirurgia, 2018.

15. JI C, et al. Is Elective Cancer Surgery Safe During the COVID-19 Pandemic? World Journal of Surgery, 2020; 44(10):3207-3211.

16. MANRIQUE BT, et al. Segurança do paciente no centro cirúrgico e qualidade documental relacionadas à Infecção cirúrgica e à hospitalização. Acta Paul Enferm. 2015; 28(4):355-60

17. MARTINS FZ, DALL'AGNOL CM. Centro cirúrgico: desafios e estratégias do enfermeiro nas atividades gerenciais. Revista Gaúcha de Enfermagem, 2016.

18. MARTINS T, et al. Fatores de risco para infecção do sítio cirúrgico em cirurgias potencialmente contaminadas. Texto Contexto Enfermagem, 2018

19. MORAES ACFG. Dificuldades no combate de infecções em centro cirúrgico da rede pública: uma revisão de literatura. Revista de pesquisa: cuidado é fundamental. Rio de Janeiro, 2011;3(2):1889-1893.

20. PRATES CG, et al. Comparação das taxas de infecção cirúrgica após implantação do checklist de segurança. Acta Paulista de Enfermagem,2018; 31(2):116-122.

21. RIBEIRO HCTC, et al. Adesão ao preenchimento do checklist de segurança cirúrgica. Caderno Saúde Pública, 2017.

22. ROSCANI ANCP, et al. Validation of surgical checklist to prevent surgical site infection. Acta Paulista de Enfermagem, $2015 ; 28(6): 553-65$. 